\title{
A Study on Biofilm Phenotype and Antimicrobial Resistance Pattern of Clinical Isolates from Catheter Associated Urinary Tract Infection
}

\author{
Neha Haswani*, Tasmiya Katheeja and Sathya Anandam
}

Department of Microbiology, Yenepoya Medical College, university road,

Deralakatte, Mangalore-575018, Karnataka, India

*Corresponding author

\author{
A B S T R A C T
}

\begin{tabular}{|c|}
\hline Keywords \\
\hline $\begin{array}{l}\text { Catheter associated } \\
\text { urinary tract } \\
\text { infections, } \\
\text { Biofilm, } \\
\text { Tissue culture } \\
\text { plate method. }\end{array}$ \\
\hline Article Info \\
\hline $\begin{array}{l}\text { Accepted: } \\
\text { 15 October } 2017 \\
\text { Available Online: } \\
10 \text { December } 2017\end{array}$ \\
\hline
\end{tabular}

\section{Keywords}

urinary tract

infections,

plate method.

Article Info

Accepted:

Available Online:

10 December 2017
Biofilms associated with indwelling medical devices are a major public health concern now-a-days as they are difficult to treat with the routine antibiotics. So the present study was taken up to study the biofilm producing phenotype and antimicrobial resistance pattern of pathogens in patients with catheter associate urinary tract infection (CAUTI). Method: this prospective study with 50 urine samples from catheterized patients (indwelling urinary catheter of more than $48 \mathrm{hrs)} \mathrm{was} \mathrm{carried} \mathrm{out} \mathrm{over} \mathrm{a} \mathrm{period} \mathrm{of} \mathrm{three}$ months. Isolation and identification of the organism was followed by detection of biofilm phenotype by the tissue culture plate method. The results were analysed using SSPS 22.0 software. The most common organism isolated was E. coli (54\%), followed by Klebsiella pneumoniae (16\%). Among the total isolates, $31(62 \%)$ were positive for the biofilm phenotype. Biofilm producing strains showed relatively higher antibiotic resistance than non-producers. $71 \%$ of the biofilm producers were multidrug resistant. Our study showed a significant association between biofilm production and multidrug resistant.

\section{Introduction}

Biofilm is a sessile microbial community which is characterized by the cells having capacity to irreversibly attach to the substratum (either animate or inanimate) or to each other and being embedded in a matrix of self-produced extracellular polymeric substances (Donlan et al., 2002). Biofilms enhance the virulence of an organism, thereby rendering a less virulent organism or an avirulent microbe with huge power of being virulent ${ }^{2}$. They are currently estimated to be responsible for over $65 \%$ of nosocomial infections and $80 \%$ of all microbial infections (Romling et al., 2012).
Catheter associated urinary tract infection (CAUTI) is a common health care associated infection throughout the world, occurring due to one common reason of magnanimous usage of indwelling urinary catheters teamed with inappropriate antibiotic use (Majumder et al., 2014). The mechanism of such infections is mostly due to the inert nature of the catheter material which is susceptible to microbial colonization and formation of biofilm, thus enhancing the pathogenic effects (Trautner and Darouiche, 2004). Medically, biofilms decrease the antimicrobial susceptibility and render treatment difficult. 
CAUTIs are associated with recurrences and complication, thus posing a major health concern; eventually leading to urosepsis and death if not treated ${ }^{7}$. Also, due to extreme closeness of microbial cells in a biofilm, the spread of antimicrobial resistance hastens up due to plasmid exchange.

The present study was conducted to detect biofilm production and their antimicrobial susceptibility pattern in pathogens isolated from cases of CAUTI.

The main aim and objectives of this study includes the microbial profile of catheter associated urinary tract infections. To study the biofilm production in the isolates from catheter associated urinary tract infections. And also to study the antimicrobial resistance pattern of the isolates from catheter associated urinary tract infections.

\section{Materials and Methods}

Study design: Prospective

Ethical clearance: The study was approved by the institutional ethical committee

Place of study: Tertiary care hospital, Mangalore, Karnataka

Duration of the study: 3 months (June $2016-$ August 2016)

Sample size: 50 non repetitive samples.

\section{Inclusion criteria}

Urine samples received with appropriately filled test requisition form (TRF's).

Samples which yielded monomicrobial growth

\section{Exclusion criteria}

Samples with polymicrobial growth. Repeat sample from the same patient.

\section{Statistical analysis}

Descriptive data with frequency in numbers was collected and was statistically evaluated using the software SPSS 22.0. A chi square test was done to establish an association between biofilm production and Multidrug resistance.

\section{Isolating the pathogen}

The samples were inoculated on Blood agar, MacConkey's agar and Cysteine lactose electrolyte deficient medium using calibrated loops of $4 \mathrm{~mm}$.

Antibiotic sensitivity was performed on Muller Hinton agar plates by the Kirby Bauer disc diffusion method. The isolates were stored in nutrient agar in preservation vials at $2-8^{\circ} \mathrm{C}$ until further processing and detection of biofilms.

\section{Biofilm production}

Biofilm producing capacity of the isolate was detected by the tissue culture plate method as described by Mathur et al., (2006).

The organisms preserved in nutrient broth were inoculated in brain heart infusion broth (BHI) with $2 \%$ sucrose. The broth was incubated at $37^{\circ} \mathrm{C}$ for $18-24$ hours. The broth culture was then diluted 1:100 with fresh brain heart infusion broth with $2 \%$ sucrose.

Individual wells of sterile 96 well flat bottom polystyrene tissue culture plates were filled with $200 \mu 1$ of diluted cultures. The tissue culture plates were then incubated at $37^{\circ} \mathrm{C}$ for 24 hours. After incubation content of each well was removed by gently tapping the plates on a surface. The wells were washed 4 times with $0.2 \mathrm{ml}$ of phosphate buffer saline $(\mathrm{pH}$ 7.2) to remove the free floating "planktonic" bacteria. Biofilms formed by adherent sessile 
organisms in plate were fixed with $2 \%$ sodium acetate and stained with $0.1 \% \mathrm{w} / \mathrm{v}$ of crystal violet. Excess stain was removed by thoroughly washing the tissue culture plate with deionized water three times and then the plates were kept for drying. Optical densities (OD) of stained adherent bacteria were determined with an ELISA reader at wavelength of $570 \mathrm{~nm}$. These OD values were considered as an index of bacteria adhering to surface and forming biofilm.

\section{Results and Discussion}

Out of 50 patients $39(78 \%)$ were male and 11 $(22 \%)$ were female.

Most common age group was 25 to 40 years which made up 60\% (30 out of 50) of total study population. Diabetes mellitus was found to be a co-morbid condition associated with $48 \%(n=24)$ of the patients. E. coli was the commonest organism seen in $54 \%(n=27)$ of samples, followed by Klebsiella pneumoniae (16\%), Enterococcus feacalis (12\%), Pseudomonas aeruginosa (10\%), Acinetobacter baumannii and Coagulase Negative Staphylococcus (4\% each) (Table $1)$.

$62 \%(\mathrm{n}=31)$ of the strains were positive for in vitro biofilm production. With $70 \%$ of the isolates being positive for the biofilm phenotype, E. coli was a significant biofilm producer in this study. It was followed by Klebsiella (63\%), Pseudomonas (60\%), Acinetobacter (50\%) and Enterococcus (50\%). All isolates of Coagulase Negative Staphylococci (CONS) were negative for in vitro biofilm formation (Table 2).

The antimicrobial resistant pattern of biofilm producers and non-producers was compared. In the present study, $80 \%$ of the total isolates were resistant to cotrimoxazole, of which 25 were biofilm producers and 15 were non biofilm producers. Similarly $80 \%$ isolates were resistant to Nalidixic acid, of which 27 were biofilm producers and 13 were non biofilm producers.

Among the biofilm producers maximum resistance was observed for Nalidixic acid (87\%), followed by Norfloxacin (81\%), Ampicillin (77\%), Ceftotaxime (74\%) (Table $3)$.

Multidrug resistance is an inherent feature of biofilm producing organisms, which further enhances their virulence. Out of the 31 biofilm producers, $22(71 \%)$ of the isolates were multidrug resistant.

There was a strong correlation between biofilm production and multidrug resistance with $\mathrm{p}$ value $<0.0001$ (Table 4).

Table.1 Microbial profile of catheter associated urinary tract infections $(n=50)$

\begin{tabular}{|l|l|}
\hline Organisms & No of isolates (\%) \\
\hline E.coli & $27(54 \%)$ \\
\hline Klebsiella pneumoniae & $8(16 \%)$ \\
\hline Pseudomonas aeruginosa & $5(10 \%)$ \\
\hline Acinetobacter baumannii & $2(4 \%)$ \\
\hline Enterococcus faecalis & $6(12 \%)$ \\
\hline CONS & $2(4 \%)$ \\
\hline
\end{tabular}


Table. 2 Screening for biofilm production $(\mathrm{n}=50)$

\begin{tabular}{|l|l|l|l|}
\hline \multirow{2}{*}{ Bacterial organisms } & \multicolumn{2}{|l|}{ Biofilm producers (\%) } & \multirow{2}{*}{ Total } \\
\cline { 2 - 3 } & Positive & Negative & \\
\hline E. coli & $19(70 \%)$ & $8(30 \%)$ & 27 \\
\hline Klebsiella pneumoniae & $5(63) \%$ & $3(37 \%)$ & 8 \\
\hline Pseudomonas aeruginosa & $3(60) \%$ & $2(40 \%)$ & 5 \\
\hline Acinetobacter & $1(50) \%$ & $1(50 \%)$ & 2 \\
\hline Enterococcus feacalis & $3(50) \%$ & $3(50 \%)$ & 6 \\
\hline CONS & $0(0 \%)$ & $2(100 \%)$ & 2 \\
\hline Total & $31(62 \%)$ & $19(38 \%)$ & 50 \\
\hline
\end{tabular}

Table.3 Antimicrobial resistance pattern of biofilm and non-biofilm producers

\begin{tabular}{|l|l|l|l|}
\hline \multirow{2}{*}{ Antibiotics } & \multicolumn{3}{|c|}{ Resistance } \\
\cline { 2 - 4 } & $\begin{array}{l}\text { Biofilm positive } \\
(\mathrm{n}=31)\end{array}$ & $\begin{array}{l}\text { Biofilm negative } \\
(\mathrm{n}=19)\end{array}$ & $\begin{array}{l}\text { Resistance of all } \\
\text { isolates (n = 50) }\end{array}$ \\
\hline Ampicillin (10 $\boldsymbol{\mu g})$ & $24(77) \%$ & $9(47) \%$ & $33(66) \%$ \\
\hline Amikacin(30 $\mathbf{~ g g )}$ & $9(29) \%$ & $2(11) \%$ & $11(22) \%$ \\
\hline Cefotaxime(30 $\boldsymbol{\mu g})$ & $23(74) \%$ & $8(42) \%$ & $31(62) \%$ \\
\hline Cotrimoxazole & $25(81) \%$ & $15(78) \%$ & $40(80) \%$ \\
\hline Norfloxacin(10 $\boldsymbol{\mu g})$ & $25(81) \%$ & $11(58) \%$ & $36(72) \%$ \\
\hline Gentamycin $(\mathbf{1 0} \boldsymbol{\mu g})$ & $14(45) \%$ & $8(42) \%$ & $22(44) \%$ \\
\hline Nalidixic acid(30 $\mathbf{\mu g})$ & $27(87) \%$ & $13(68.4) \%$ & $40(80) \%$ \\
\hline Nitrofurantion $\mathbf{( 3 0 0} \boldsymbol{\mu g})$ & $18(58) \%$ & $6(32) \%$ & $24(48) \%$ \\
\hline
\end{tabular}

Table.4 Co-relation between multidrug resistant phenotype and Biofilm production

\begin{tabular}{|l|c|c|c|}
\hline Drug resistance & Biofilm producer & Non biofilm producer & p.value \\
\hline MDR & 22 & 3 & \\
\hline Non MDR & 09 & 16 & $\mathrm{p}<0.0001$ \\
\hline
\end{tabular}

In the present study, E. coli was the predominant gram negative pathogen isolated, accounting for almost $54 \%$ of the total isolates (27 out of 50); followed by Klebsiella pneumoniae (16\%), Pseudomonas aeruginosa (10\%)and Acinetobacter baumannii (4\%). Amongst the gram positive isolates Enterococcus feacalis was most common being $12 \%$ of the total isolates, followed by Coagulase negative Staphylococcus (4\%).

Similar findings of E. coli being the commonly associated pathogen with CAUTI have been reported by Deotale et al.,(2015) $(51.4 \%)$ and Pramodhini et al.,(2012) (70\%).

The present study reveals $62 \%$ (31 out of 50) of the strains were in vitro positive for biofilm production. Similar results were observed by Niveditha et al., (2012), who reported $60 \%$ of their strains to be positive for in-vitro biofilm production. On the contrary Abdullah et al., ${ }^{4}$ reported only $43.3 \%$ of the isolates to be biofilm producers In our study, significant proportion of biofilm formation was seen in E.coli (70\%), followed by Klebsiella (63\%), 
Pseudomonas (60\%) and Acinetobacter and Enterococcus which were $50 \%$ each whereas coagulase negative staphylococci (CONS) did not display biofilm positive phenotype.

Similar findings have been reported by Niveditha et al.,(2012). Sharma et al.,(2012) reported a similar rate of biofilm production in E. coli $(67.5 \%)$. Ponnuswamy et al., reported $72 \%$ of $E$. coli strains having biofilm positive phenotype.

It is a well-known fact that antimicrobial resistance is an innate feature of bacterial biofilms that may further lead to treatment failure. Keeping this in mind, we studied the antibiotic resistance pattern of all uropathogens isolated and found the isolates positive for biofilm formation to have a higher drug resistance as compared to the non-biofilm producing isolates. In the present study, resistant to antibiotics like ampicillin, cefotaxime, norfloxacin and nalidixic acid was noted in $78 \%, 76 \%, 70 \%$ and $87 \%$ of the total biofilm producing isolates; which is much higher than non-biofilm producers.

About $71 \%$ of the biofilm producers were found to be multidrug resistant. Similar findings have been documented by Pramodhini et al., (2012), who reported about $80 \%$ of their isolates to be multidrug resistant. In our study we found a very strong correlation between biofilm production and multidrug resistant pattern with $\mathrm{p}$ value < 0.0001 .

E. coli was the most common isolate and $70 \%$ of them were found to be biofilm producers. Biofilm producing isolates displayed higher degree of antibiotic resistance as compared to the biofilm non producers. We also observed a significant correlation between biofilm production and multidrug resistance.

CAUTIs have a definite economical concern as it increases the hospital stay as well as the cost of medication. Judicious use of antibiotics and good catheter care can go a long way in reducing the burden of Catheter associated urinary tract infections.

\section{Acknowledgement}

Special thanks to ICMR for granting the ICMR STS 2016 (ID: 2016-00206) scholarship for this project.

\section{References}

Donlan RM and Costerton JW. 2002. Biofilms: Survival mechanisms of clinically relevant microorganisms. Clin. Microbiol. Rev., 15(2): 167-193.

Intesar N. Khelkal. Biofilm formation and antibiotic resistance of uropathogenic $E$. coli isolated from urinary tract of catheterized patients. Journal of Genetic and Environmental Resources Conservation, 2015, 3(1):66-73.

Majumder, M.I., Ahmed, T., Hossain, D., Ali, M., Islam, B. and Chowdhury, N.H. Bacteriology and antibiotic sensitivity patterns of urine and biofilm in patients with indwelling urinary catheter in a tertiary hospitals in Bangladesh. J. Bacteriol. Parasitol., 2014, 5(3): 191196.

Nermeen Mahmoud Ahmed Abdallah et al., Biofilm forming bacteria isolated from urinary tract infection, relation to catheterization and susceptibility to antibiotics. International Journal for Biotechnology and Molecular Biology Research., 2011, 2(10), 172-178.

Niveditha $\mathrm{S}$ et al., The isolation and the biofilm formation of uropathogens in the patients with catheter associated urinary tract infections (UTIs). J. Clin. Diag. Res. 2012, 6(9): 1478.

Poovendran Ponnusamy et al., In vitro biofilm formation by uropathogenic 
Escherichia coli and their antimicrobial susceptibility pattern. Asian Pacific Journal of Tropical Medicine, 2012, 210-213.

Ramya Amuthamani et al., 2017. Biofilm Producing Uropathogens and Drug Resistance: Dual Foe for Patients on Urinary Catheter. Int.J.Curr.Microbiol. App. Sci., 6(5): 326-330.

Romling, U. and Balsalobre C. Biofilm infections, their resistance to therapy and innovative treatment strategies. J. Int. Med., 272, 2012(6): 541-561.

Sharma $\mathrm{M}$ et al., Biofilm production in uropathogenic Escherichia coli. Indian. J. Pathol. Microbiol., 2009, 52(2): 294.

Subramanian Pramodhini et al., 2012. Antibiotic resistance pattern of biofilm forming uropathogens isolated from catheterized patients in Pondicherry, India. Australasian Medical Journal, 5, 7, 344-348.

T Mathur et al., 2006. Detection of biofilm formation among the clinical isolates of staphylococci: An evaluation of three different screening methods. Indian journal of medical microbiology, 24(1), 25-29.

Trautner BW and Darouiche RO. 2004. Role of biofilm in catheter associated urinary tract infection. Am. J. Infect. Control, 32: 177-118.

Deotale, V. S. et al., 2015.Correlation between biofilm formation and highly drug resistant uropathogens (HDRU). Int J Cur Res Rev, 7(2), 61-65.

\section{How to cite this article:}

Neha Haswani, Tasmiya Katheeja and Sathya Anandam. 2017. A Study on Biofilm Phenotype and Antimicrobial Resistance Pattern of Clinical Isolates from Catheter Associated Urinary Tract Infection. Int.J.Curr.Microbiol.App.Sci. 6(12): 1972-1977. doi: https://doi.org/10.20546/ijcmas.2017.612.226 